\title{
The Expression of Architectural Decoration Engineering Design Effect Based on Computer Aided Software
}

(Shaanxi Energy Vocational and Technical College, Xianyang, Shaanxi 712000)

\section{Introduction}

Before the development and analysis of the building decoration works under the computer aided drawing software, we must fully understand the development course of the origin of the architectural decoration discipline. In the eighties of last century, with the deepening of China's reform and opening up and economic development, people's pursuit of the United States continues to rise, especially in the living and living environment of the pursuit of beauty. Which appeared to the United States as the scale of the architectural design. At the same time, the pace of development in the field of society to accelerate the pace of almost all of the products need to be carefully designed to meet the market, designed to become a very popular industry and almost all of the public products need to design ${ }^{[1]}$. For architectural decoration is the indoor collaborative outdoor environmental art atmosphere, the embodiment of the atmosphere, the specific architectural design needs to meet the needs of indoor and outdoor living environment at the same time, start all aspects of the design and planning work, including indoor and outdoor forms, walls, doors, lighting, home furnishings, decorating materials, home appliances, etc. design and planning.

As a professional architectural design staff requirements must master the basic computer graphics software operation and drawing skills, whether it is home improvement or tooling, are required to design the first design renderings and construction drawings. Drawings are often done with the following computer graphics software: AUTOCAD, Photoshop, 3DMAX, CorelDRAW, etc. ${ }^{[2]}$. Based on the above-mentioned integrated graphics editing, graphics drawing computer graphics software functions, can be based on architectural design drawings to meet the composition of the composition and the actual reflection of the concept, for this reason, most of the building decoration staff will use the computer graphics software to carry out the design work. Therefore, computer-aided software for building decoration engineering design has an important impact.

\section{Plan design highlights the in- tuitive and creative advantages}

For example, the characteristics of the interior style of Jane Europe, whether it is the whole house or the local people are elegant and impressed. Jane Europe home style most use light or wood color home and meet the simple and delicate style of curtains, wallpaper, carpets and furniture, and so all reflect the traditional European historical impression and cultural charm, Jane European home style does not use the traditional European classical texture finishes, learn the beauty of modern decoration, weaken the European style lines, the subject highlights simple, ful

\begin{abstract}
In the 21st century, the domestic economic level has been improved rapidly. At the same time, it has made great progress in the field of building decoration engineering. The building decoration industry has the characteristics of a knowledge-intensive, highly comprehensive and large-scale investment. With the progress of society, architectural decoration design tools have also been with the progress in the building decoration engineering computer-aided design of a wide range of popular applications, change the original product design and production model, the formation of a new architectural decoration engineering design model, which is the biggest change in the decoration industry. For example, the computer technology used in architectural design work, the use of computer technology to collect the corresponding reference materials and computer-aided design to replace the traditional hand-painted and so on. Computer graphics software has the advantages of reasonable layout, easy modification and convenient storage. Thus, computer-aided software is applied in the field of architectural decoration engineering design, which drives the design of building decoration engineering to a higher level. The application of computer aided software can fully reflect its important role in the design of architectural decoration engineering.
\end{abstract}

Key words: Computer aided software; architectural decoration works; decorative design

Published on 15th July, 2017 
ly demonstrated the noble and elegant style of home ${ }^{[3]}$. The theme of the architectural design of the architectural style is Jane European style, through Abstract: In the 21 st century, the domestic economic level has been improved rapidly. At the same time, it has made great progress in the field of building decoration engineering. The building decoration industry has the characteristics of a knowledge-intensive, highly comprehensive and largescale investment. With the progress of society, architectural decoration design tools have also been with the progress in the building decoration engineering computer-aided design of a wide range of popular applications, change the original product design and production model, the formation of a new architectural decoration engineering design model, which is the biggest change in the decoration industry. For example, the computer technology used in architectural design work, the use of computer technology to collect the corresponding reference materials and computer-aided design to replace the traditional hand-painted and so on. Computer graphics software has the advantages of reasonable layout, easy modification and convenient storage. Thus, computer-aided software is applied in the field of architectural decoration engineering design, which drives the design of building decoration engineering to a higher level. The application of computer aided software can fully reflect its important role in the design of architectural decoration engineering.

the analysis of the elegant style of luxury, revealing the honor of the design performance, reflecting the human quality of the living environment for the pursuit of the pursuit of an artistic sense of life attitude ${ }^{[4]}$.

To Simplistic European style architectural design for the analysis of the theme, the main study of a combination of ancient Europe noble and elegant, modern simple, generous, elegant design style of the apartment ${ }^{[5]}$.
This case to a European-style interior design as an example to start a specific analysis, living room, kitchen and other design as shown below.
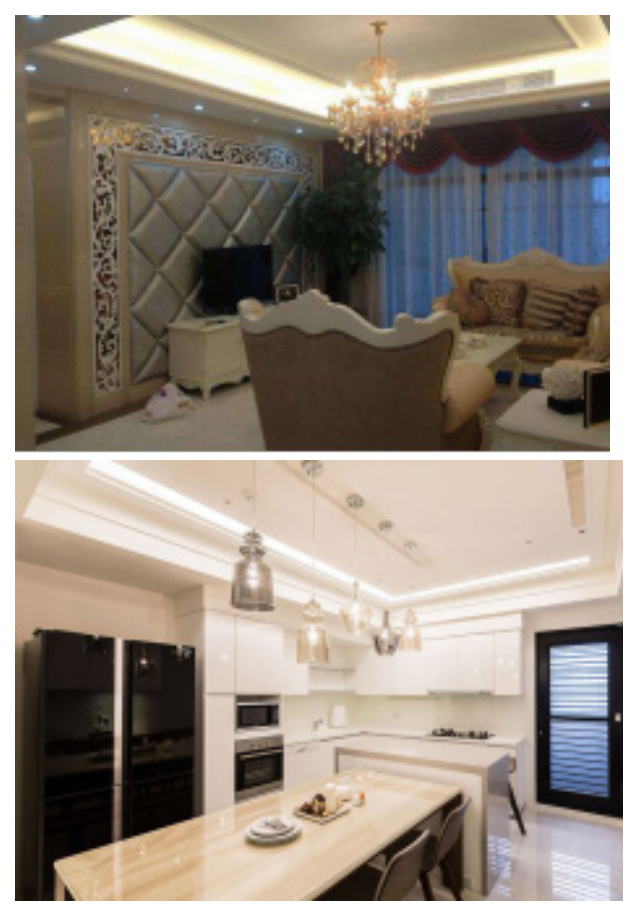

Figure 1 Living room Figure 2 Kitchen
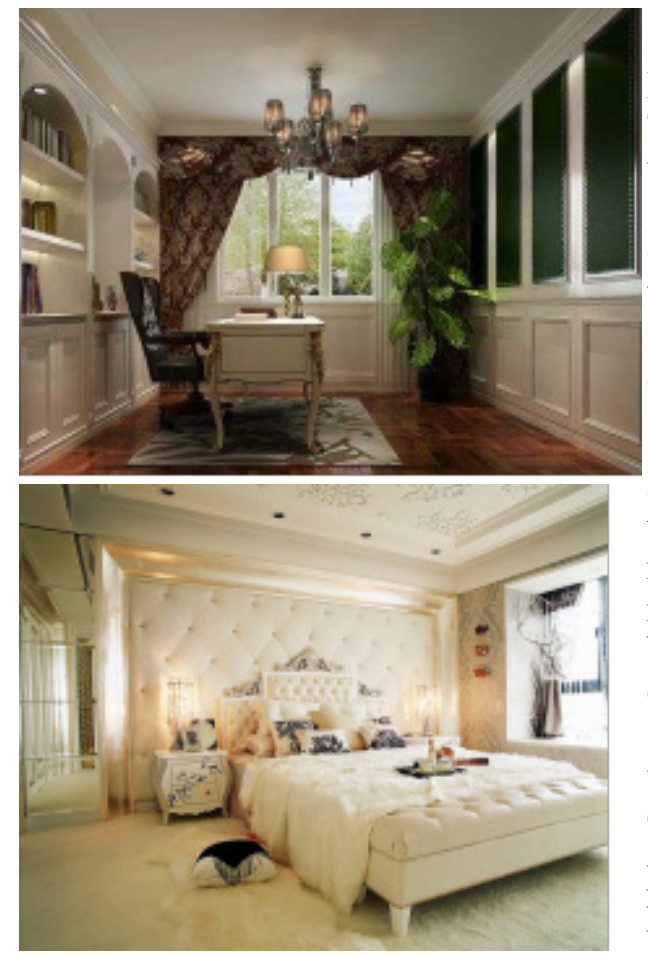

Figure 3 Study room Figure 4 Bedroom

\section{Computer-aided software perfor- mance}

2.1 Layout aspects
Based on the overall departure, the layout of the apartment layout in addition to meet the needs of the head of life, in the overall interior decoration with European-style marble as the main tone, to strengthen the indoor space of the atmosphere and texture at the same time to create a warm home feeling.

The top of the living room with elegant plaid gypsum board ceiling, a ceiling set up the atmosphere of the elegant chandelier as a foil, interior design of the floor window, and selected in line with Jane Europe's elegant curtain cover, the interior to create a full comfortable dripping space sensory ${ }^{[6]}$.

TV backdrop is the choice of simple beige single-sided wall and the rear side of the transparent glass echoes the background wall before the layout of the black and white TV and the overall harmony, floor windows on both sides of the wall brush white latex paint, reflecting its texture. The ground with a wooden floor to create a warm home feeling.

The sofa is the use of beige cloth European sofa placed in front of the $\mathrm{TV}$, the sofa placed four brown velvet sofa pad, the TV backdrop with soft leather nail decorated, surrounded by modern methods to simulate vines decorated to limit decoration, fully embodies the sense of space and modern sense ${ }^{[7]}$.

The restaurant and the kitchen are in open form, beautifully set in the wine cabinet, connected to the restaurant to form a space open, reflecting the public area of open and bright, constitute a modern visual center.

\subsection{Kitchen pattern}

The kitchen table is placed with green plants, dotted with the whole kitchen pattern. The kitchen is equipped with a transparent glass table and wooden chairs, simple and generous with a sense of space and modern fashion sense, while the wooden chairs and chairs to fully demonstrate the simple European style restaurant culture ${ }^{[8]}$. The kitchen wall with a strong reflection of the tiles, hanging cabinet with glass panels, the formation of a 
similar mirror effect, visually expand the kitchen interior area, while enhancing the kitchen transparent and clear features ${ }^{[9]}$.

The kitchen to take the open pattern, the kitchen vision space expanded, increased the head of the family and their relatives and friends cooking fun, to promote family cooking communication and interaction, bring extra fun ${ }^{[10]}$. Open kitchen requires its cupboard with a high degree of storage function, and vice versa scattered kitchenware, kitchen electric kitchen view, do not meet the simple requirements. At the same time kitchen, kitchenware with white-based color to better match the overall home color and decoration style.

\subsection{Study room style}

Study room style to maintain the overall coordination, but also to meet the following four elements:

(1) The study room requires its lighting and adequate lighting, so the computer desk or desk must be placed in the sunny and adequate light in the study room. Writing desk that people in sitting position cannot have a backlight.

(2) The study room requires its sound insulation measures complete. The choice of sound insulation materials as a study room's wall or facade design to ensure that the owner can the study out from the external environment noise interference and to maintain silent of the study room.

(3) Placed on the desk 2 to 3 pots of green plants, the back of the desk wall to do arched bookcases cut off to meet the needs of the study at the same time, fit the overall space Jane European decorative style, which fully reflects the study of the elegant.

(4) The study room is for study and workplace, which needed to keep the study room to be neat. Do not mess up with items and keep items in place.

\subsection{Bedroom space}

Bedroom space can be small, but must be warm and livable, for this choice beige and white-based color tone, the form is used the bay win- dow, to meet the owner to enjoy the outside scenery ${ }^{[11]}$. Bedroom with white-based European style wardrobe, wardrobe pattern together in the bedroom wall pattern similar to the full display of ancient European classical elegance. Whether it is bedside pattern, bedside lamp color theme, the bed was the shop pattern must be consistent with Jane Europe's simple tone style, while the above-mentioned fine workmanship, simple lines, reflecting the combination of modern and classical design of the United States. Bedroom with spotlights and table lamps for the atmosphere to create, indoor wall finishes for the yellow decorative painting. Through the details of the design and processing, and all reflect the bedroom comfortable and comfortable ${ }^{[12]}$.

\section{Commonly used computer-aided software co-performance advan- tages and development trends}

\subsection{Computer-aided software perfor- mance advantages}

The use of computer-aided software such as: AUTOCAD computer-aided design, 3DMAX 3D graphics software, Vray renderer, Photoshop, CorelDRAW plane image processing software, Premiem video editing software, through the integration of the software painting function, design a comprehensive architectural decoration design, The use of the computer graphics software have their own strengths, and fully designed a good design and construction plans, driving the building decoration industry Xiangrong development ${ }^{[13]}$. The large number of computer graphics software emerged, replacing the traditional hand-painted construction drawings, and repeatedly modified a lot of time-consuming problems, so that the new computer graphics software to complete the graphics creation, graphics editing operations, and can meet the many revised Features. At the same time two-dimensional graphics editing can be converted into three-dimensional modeling, to carry out a series of operations to complete the final design product performance renderings. To help designers better reflect their own creativity and the application of computer software, the rapid upgrade of the drawing design efficiency.

3.2 The development trend of computer aided software

Design is the human material civilization and spiritual civilization bridging viaduct, people are constantly designed to change life, change the world, greatly upgrading the quality of human life. Domestic interior design started late, the cumulative development time of about 30 years ${ }^{[14]}$, but the domestic interior design development speed, and the gap between the developed countries significantly shortened [15]. At the same time computer technology to the architectural design industry has brought unprecedented momentum to enhance the design efficiency and design simplicity, computer software applications under the innovative design products continue to be created, greatly stimulating the rapid development of the building decoration industry, while based on computer operation can be completed many times to design changes, creative design works design performance is particularly prominent, computer-aided software for the traditional architectural decoration design has brought innovative changes, but also well cater to the growing public building decoration design needs. Computer-aided design of the design of the building decoration works more intuitive, designers and owners to communicate more direct, experience the effect is significant, has become an indispensable industry auxiliary tool.

\section{Conclusion}

The use of computer software for architectural decoration design has become a mainstream form of social practice, in practice, computer-assisted and traditional hand-painted can complement each other, complement each other. Through the development of computer-aided software, the architectural design of the future expression will also become more and more intelligent. Undeniable, the use of computer software design will also extend a lot of problems, which requires decorative design practitioners and software developers continue to improve and improve the comput- 
er-aided design experience, upgrade computer technology, with the development of the eyes of computer-aided design software development and application.

\section{References}

[1] Ma Aiping. Analysis of Computer Aided Design Software in the Interior Design of the Application [J]. Coastal enterprises and technology.2012 (07): 23-25.

[2] Li Guyu. Application of 3DMAX Software in Interior Design [J]. Modern Decoration (Theory).2016 (12): 215.

[3] Xing Wei. Computer Simulation Analysis in the Green Building Design Application [J]. Automation and Instrumentation.2014 (09): 99-100 + 103.

[4] Du Chunling. Design and Application of Computer Aided System for Building Construction Measurement [J]. Automation and Instrumentation.2014 (12): 121-123.

[5] HUANG Wei-wei. Study on Design of Building Space Environment
Based on Virtual Reality Technology $[\mathrm{J}]$. Automation and Instrumentation.2010 (04): 31-33.

[6] Lu Jia. Interior Design 3DMAX Software Application [J]. Electronic Technology and Software Engineering.2013 (21): 99.

[7] Luan Chen, Li Hong. Research on the Application of Computer in Interior Design [J]. Vocational Technology.2014 (01): 93.

[8] Wang Xu. On the Development $\mathrm{f}$ Computer Architecture Design Animation - To Interior Design as an Example $[\mathrm{J}]$. Technology and Education (late).2013 (01): $82+86$.

[9] Chen Huangqing. Environmental Art Design In The Use And Development Of Digital Technology [D]. Anhui University of Engineering.2012

[10] Xie Dong. Environmental Art Design Hand-Painted Performance Techniques [D]. Guangdong University of Technology.2013

[11] Yin Ying. Talking about the Application of PHOTOSHOP Software in Architectural Decoration Design [J]. Popular Literature.2014 (09): 100.

[12] Xu Huachun, Zhu Zongjiu. Based on STM32 Family Intelligent Home Design [J]. Small and medium-sized enterprise management and technology (late).2017 (01): 150151.

[13] Lin Yinda .AutoCAD and SketchUp Combined with Architectural Design Drawing [J]. Art Education Research.2014 (11): 68-69

[14] ZHANG Fei, HONG Miao, HAN Rui. Application of Computer Technology in Architectural Decoration Design [J]. Jiangxi Building Materials.2017 (02): 41-42.

[15] Ning Bo. On the Architectural Decoration Design of the Color Mix And Match Scheme [J]. Heilongjiang Science and Technology Information.2017 (10): 183. 